\title{
Ultrasound contrast-enhanced imaging and in vitro antitumor effect of paclitaxel-poly(lactic-co-glycolic acid)-monomethoxypoly (ethylene glycol) nanocapsules with ultrasound-targeted microbubble destruction
}

\author{
JING MA ${ }^{1,2^{*}}$, LING XI XING ${ }^{1 *}$, MING SHEN $^{3}$, FAN LI $^{1}$, MING JIE ZHU ${ }^{3}$, LI FANG JIN ${ }^{1}$, \\ ZHAOJUN LI ${ }^{1}$, FENG GAO ${ }^{1}$, YIJIN SU ${ }^{1}$, YOU RONG DUAN ${ }^{3}$ and LIAN FANG DU ${ }^{1}$ \\ ${ }^{1}$ Department of Ultrasound, Shanghai First People's Hospital Affiliated to Shanghai Jiao tong University School of Medicine, \\ Shanghai 200080; ${ }^{2}$ Department of Cardiovascular Ultrasound, Shanghai East Hospital Affiliated to Tong ji University, \\ Shanghai 200120; ${ }^{3}$ Cancer Institute, Renji Hospital Affiliated to Shanghai Jiao tong University School of Medicine,
} Shanghai 200032, P.R. China

Received January 10, 2014; Accepted October 2, 2014

DOI: $10.3892 / \mathrm{mmr} .2014 .3072$

\begin{abstract}
A combination of diagnostic and therapeutic ultrasound (US) techniques may be able to provide the basis of specific therapeutic protocols, particularly for the treatment of tumors. Nanotechnology may aid the progression towards the use of US for tumor diagnosis and targeted therapy. The current study investigated in vivo and in vitro US contrast imaging using nanocapsules (NCs), and also US and US-targeted microbubble destruction (UTMD) therapy using drug-loaded NCs for pancreatic cancer in vitro. In the current study, the NCs were made from the polymer nanomaterial poly(lactic-co-glycolic acid)-monomethoxypoly(ethylene glycol) (PLGA-mPEG), encapsulated with paclitaxel (PTX), to create PTX-PLGA-mPEG NCs. The PTX-PLGA-mPEG NCs were used as a US contrast agent (UCA), which produced satisfactory US contrast-enhanced images in vitro and in vivo of the rabbit kidneys, with good contrast compared with lesions
\end{abstract}

Correspondence to: Professor Lian Fang Du, Department of Ultrasound, Shanghai First People's Hospital Affiliated to Shanghai Jiao tong University School of Medicine, 85 Wu Jin Road, Shanghai 200080, P.R. China

E-mail: du_lianfang@163.com

Professor You Rong Duan, Cancer Institute, Renji Hospital Affiliated to Shanghai Jiao tong University School of Medicine, $2200 \mathrm{Xie}$ Tu Road, Shanghai 200032, P.R. China

E-mail: yrduan@shsci.org

*Contributed equally

Key words: nanocapsules, paclitaxel-poly(lactic-co-glycolic acid)-monomethoxy poly(ethylene glycol), ultrasound contrast-enhanced imaging, ultrasound-targeted microbubble destruction, antitumor in the peripheral regions. However, clear contrast-enhanced images were not obtained using PTX-PLGA-mPEG NCs as a UCA, when imaging the superficial pancreatic tumors of nude mice in vivo. Subsequently, fluorescence and flow cytometry were used to measure the NC uptake rate of pancreatic tumor cells under various US or UTMD conditions. An MTT assay was used to evaluate the efficiency of PTX and PTX-PLGA-mPEG NCs in killing tumor cells following 24 or $48 \mathrm{~h}$ of US or UTMD therapy, compared with controls. The specific US or UTMD conditions had been previously demonstrated to be optimal through repeated testing, to determine the conditions by which cells were not impaired and the efficiency of uptake of nanoparticles was highest. The current study demonstrated high cellular uptake rates of PLGA-mPEG NCs and high tumor cell mortality with PTX-PLGA-mPEG NCs under US or UTMD optimal conditions. It was concluded that the use of NCs in US-mediated imaging and antitumor therapy may provide a novel application for US.

\section{Introduction}

Ultrasound (US) imaging has been a primary choice for the diagnosis and evaluation of tumors, as it is safe, a real-time measurement, low-cost and portable. The wide use of US contrast agents (UCAs), which may enhance the comparison between lesions and surrounding tissue, has greatly improved the resolution and sensitivity of clinical US imaging (1). Micro-sized UCAs cannot pass through the vascular endothelium, so are consequently regarded as blood pool tracers, nano-scale UCAs however, are able to pass through gaps in the vascular endothelium into tumor mesenchyma. As a result, much cancer therapy research has focused on drug-loaded nanoparticles (NPs). A number of antitumor drugs, including cisplatin (2), mitoxantrone (3), DNA and siRNA (4) have been successfully embedded into NPs, which may provide protection from direct degradation by nucleases in vivo. In addition, NPs may facilitate drug uptake into target cells or tissues via 
an endocytic pathway (5). Previously, studies have undertaken investigations to assess the treatment of pancreatic cancer by drug-loaded NPs (6). However, the results obtained suggested that the efficiency of drug-loaded NP uptake into the tumor tissues remained low. US-targeted microbubble destruction (UTMD) may be an effective method to facilitate NP uptake into various types of tumor tissue in vivo, due to an alteration in the permeability of the vasculature and cell membrane (7-10). The aims of the current study were as follows: (i) To evaluate the use of US contrast imaging with a novel NC in vivo and in vitro; and (ii) to establish the effectiveness of US and UTMD in promoting the uptake of this paclitaxel-loaded NC (PTX-NC) in vitro into pancreatic cancer cells to induce cytotoxicity.

\section{Materials and methods}

Materials. Poly(lactic-co-glycolic acid)-monomethoxypoly(ethylene glycol) (PLGA-mPEG) (LA:GA, 8:2; PEG2000, $10 \%$ ) was obtained from Shanghai Cancer Institute (Shanghai, China). Pluronic F68 was obtained from BASF Co., Ltd. (Shanghai, China). PTX and dichloromethane were obtained from Tianjin Kaitong Chemical Reagent Co., Ltd. (Tianjin, China). Rhodamine (Rh) and fluorescein isothiocyanate (FITC) were obtained from Beijing Biosea Biotechnology Co., Ltd. (Beijing, China). SonoVue (Bracco, Milan, Italy) is a lipid-coated UCA with sulfur hexafluoride gas microbubbles (MBs), composed of $\sim 2 \times 10^{8} \mathrm{MBs} / \mathrm{ml}$, and an average diameter of 2.5-6.0 $\mu \mathrm{m}$. A total of 10 female New Zealand white rabbits (12 weeks old; average weight, $3128.54 \pm 102.32 \mathrm{~g}$ ) and 30 nude female BALB/c mice (4 weeks old; average weight, $13.87 \pm 1.92 \mathrm{~g}$ ) were supplied by the First People's Hospital Affiliated to Shanghai Jiao tong University (Shanghai, China), and all animal procedures were performed in accordance with the research protocol approved by the Animal Care and Use Committee of the hospital.

Preparation and physicochemical characteristics of the PTX-PLGA-mPEG NCs. The PTX-mPEG-PLGA NCs were prepared using the double-emulsion method (11). PTX solution (1.25 mg in $1.25 \mathrm{ml}$ methanol solution; Shanghai Baoman Biotechnology Co., Ltd., Shanghai, China) was emulsified in the PLGA-mPEG solution $(25 \mathrm{mg}$ in $1 \mathrm{ml}$ dichloromethane solution) by sonication ( $200 \mathrm{~W}, 5 \mathrm{sec}-2 \mathrm{sec}-15)$ using an ultrasonic cell disrupter (JY92-ZD, Ningbo Xinzhi Biotechnology Co., Ltd., Ningbo, China). Subsequently, $10 \mathrm{ml}$ F68 aqueous solution $(1 \mathrm{mg} / \mathrm{ml})$ was rapidly added to the first emulsion and sonicated $(200 \mathrm{~W}, 5 \mathrm{sec}-2 \mathrm{sec}-15)$. The resultant emulsions were stirred to evaporate the dichloromethane and were then lyophilized (EPSILON 2-6D; Martin Christ, Osterode am Harz, Germany). The NC sizes were measured using an H-7000 Transmission Electron Microscope (Hitachi Ltd., Tokyo, Japan). The size distribution and $\zeta$ potential of the NPs in aqueous solution was determined using a Nicomp-380ZLS $\zeta$ potential analyzer, from Particle Sizing Systems, Inc. (Port Richey, FL, USA). The drug-loading rate was calculated as the ratio of the amount of PTX encapsulated in NCs to the total amount of NCs (3 mg) initially used. The detection of drug-loading rate was completed by the Shanghai Cancer Institute (Shanghai, China).
Cell culture. Human pancreatic cancer cells (Aspc-1; Shanghai Tumor Institute, Shanghai, China) were incubated in Dulbecco's modified Eagle's medium (Gibco Life Technologies, Grand Island, NY, USA), then were maintained in $10 \%$ fetal bovine serum (Sigma, St. Louis, MO, USA), penicillin and streptomycin $(100 \mu \mathrm{g} / \mathrm{ml}$; Shanghai Baoman Biotechnology Co, Ltd.) at $37^{\circ} \mathrm{C}$ in humidified conditions with $5 \% \mathrm{CO}_{2}$. Subsequently, the pancreatic cancer cells were seeded into 6- and 96-well plates according to the different experimental conditions.

US contrast-enhanced imaging analysis of PTX-PLGA-mPEG NCs (nano-UCA) in vitro. The Philips iE33 xMATRIX US system (Philips Healthcare, Andover, MA, USA) was used with an L11-3 probe. A total of $5 \mathrm{ml}$ degassed water was poured into one 5-ml soft tube. Nano-UCA powder $(60 \mathrm{mg})$ was placed into another 5-ml soft tube and topped up with degassed water. The lids of the two tubes were then sealed tightly and the tube containing the nano-UCA solution was vibrated in order to ensure that the powder was completely dissolved prior to imaging. The outer surfaces of the soft tubes were covered with nano-UCA to prevent any air getting between the tubes and the transducer. US is attenuation in air, therefore, ultrasonic medicinal coupling gel was smeared between the tube and the transducer. US contrast images of the nano-UCA solution $(12 \mathrm{mg} / \mathrm{ml})$ were immediately collected and recorded.

US contrast-enhanced imaging analysis of PTX-PLGA-mPEG NCs (nano-UCA) in vivo. The Acuson Sequoia 512 US system (Siemens, Erlangen, Germany) and LOGIQ E9 (GE Healthcare, Wauwatosa, WI, USA) were used with the 15L8W-S and ML6-14 probes, respectively. Degassed water $(2 \mathrm{ml})$ and nano-UCA solution $(2 \mathrm{ml} ; 12 \mathrm{mg} / \mathrm{ml})$ were injected into the ear veins of the rabbits, while US contrast images of the right kidney were observed in real-time and recorded. Similarly, $1 \mathrm{ml}$ degassed water, $1 \mathrm{ml}$ nano-UCA $(12 \mathrm{mg} / \mathrm{ml})$ solution and $1 \mathrm{ml}$ SonoVue suspension were injected into the tail veins of the mice, while US contrast images of superficial pancreatic tumors in nude mice were also observed in real-time and recorded. Rabbits were euthanized via injection of $1,200 \mathrm{mg} / \mathrm{kg}$ nembutal injected into the ear vein and mice were sacrificed by decapitation.

Detection of cellular uptake of PLGA-MPEG NCs by fluorescence microscopy. Aspc-1 pancreatic cancer cells (3x105/well) were cultured in two 6-well plates and incubated for $24 \mathrm{~h}$. A therapeutic US machine (Physiomed Elektromedizin AG, Schnaittach, Germany) was used at a frequency of $1 \mathrm{MHz}$, with the optimal US conditions (power, $1 \mathrm{~W} / \mathrm{cm}^{2}$; exposure time, $60 \mathrm{sec}$; duty cycle, 20\%; SonoVue volume ratio, 1:5). The cells were divided into three groups as follows: The phosphate-buffered saline (PBS), Rh and Rh-PLGA-mPEG $\mathrm{NC}$ group. Each group was exposed to three environmental conditions: i) Control; ii) US; and iii) UTMD. The volume of solution in each well was $1 \mathrm{ml}$, with an equal volume of $\mathrm{Rh}$. Each group was evaluated and imaged using fluorescence microscopy following $5 \mathrm{~h}$ of the respective treatments.

Detection of cellular uptake of PLGA-mPEG NCs by flow cytometry. The Aspc-1 cells were divided into four groups: 
A

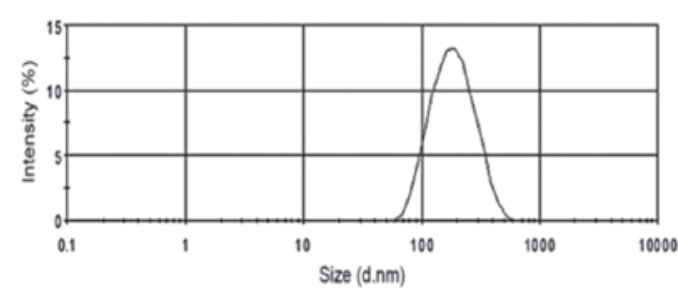

B

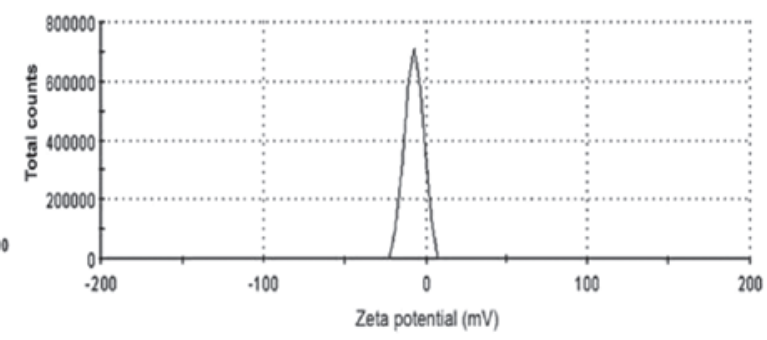

Figure 1. (A) Size distribution and (B) $\zeta$ potential of paclitaxel-poly(lactic-co-glycolic acid)-monomethoxy poly(ethylene glycol) nanocapsules.

A

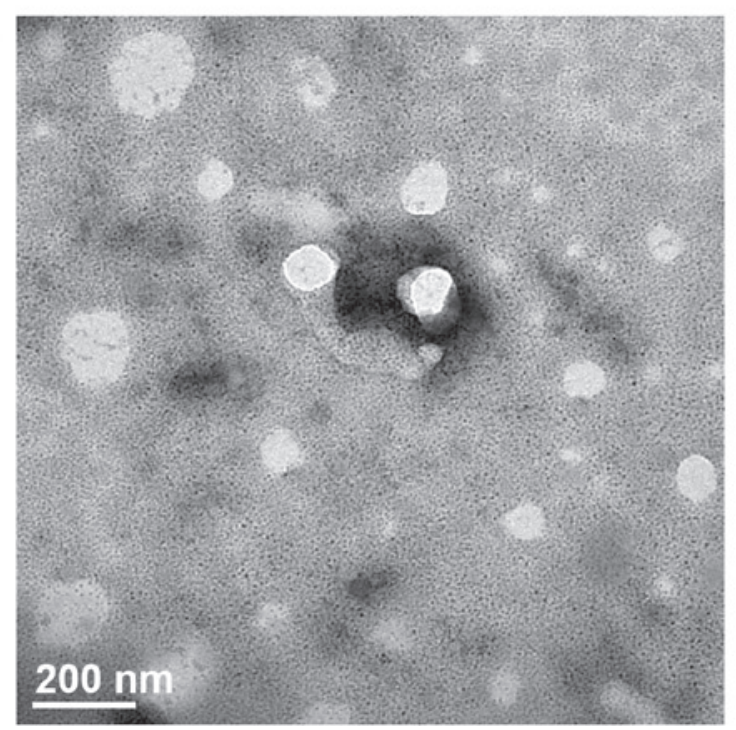

B

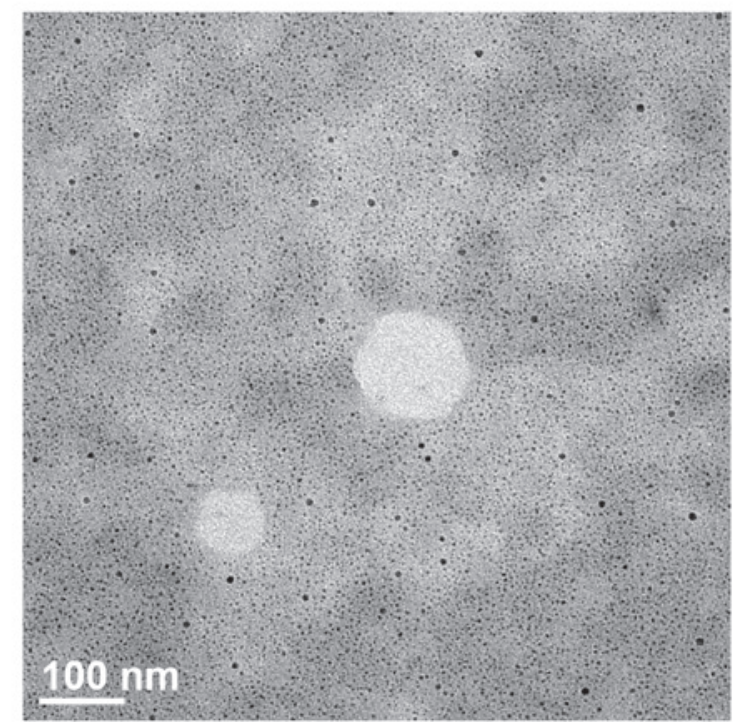

Figure 2. Transmission electron microscope images of paclitaxel-poly(lactic-co-glycolic acid)-monomethoxy poly(ethylene glycol) nanocapsules. Magnification (A) $\mathrm{x} 10,000$ and (B) $\mathrm{x} 30,000$.

The controls (Aspc-1 cells only), the FITC-PLGA-mPEG NCs, FITC-PLGA-mPEG NCs + US and FITC-PLGA-mPEG NCs + UTMD groups. The cells of each group were seeded at a density of $\sim 5 \times 10^{5}-1 \times 10^{6}$ cells/plate. The groups were exposed to the same optimal US conditions (power, $1 \mathrm{~W} / \mathrm{cm}^{2}$; exposure time, $60 \mathrm{sec}$; duty cycle, $20 \%$; SonoVue volume ratio, 1:5). The cells in each plate were washed twice with PBS subsequent to administration, and promptly harvested by trypsinization (Aladdin Industrial, Inc., Nashville, TN, USA). Subsequently, the cells were suspended in $1 \mathrm{ml}$ PBS. In the FITC-PLGA-mPEG NCs + UTMD group, $200 \mu 1$ MB solution (SonoVue) was injected into the $1 \mathrm{ml}$ PBS solution for each plate. Samples were analyzed $5 \mathrm{~h}$ subsequent to administration of NC, using a flow cytometer (EPICS XL/XL-MCL; Beckman Coulter, Miami, FL, USA).

Cellular cytotoxicity test. The cellular cytotoxicity of the NCs was determined by MTT assay. Briefly, the Aspc-1 pancreatic cancer cells $\left(1 \times 10^{5} /\right.$ well) were cultured in 96 -well plates and incubated for $24 \mathrm{~h}$. PBS solution; SonoVue dissolved in sterile saline solution (Nanjing Bianzhen Biotechnology Co., Ltd., Nanjing, China); blank PLGA-mPEG NCs; blank PLGA-mPEG NCs + US; blank PLGA-mPEG NCs + UTMD; PTX-PLGA-mPEG NCs; PTX-PLGA-mPEG NCs + US; PTX-PLGA-mPEG NCs + UTMD; and PTX were added to the cells at different concentrations, and incubated for 24 and $48 \mathrm{~h}$ at $37^{\circ} \mathrm{C}$. The optimal US conditions (power, $1 \mathrm{~W} / \mathrm{cm}^{2}$; exposure time, $60 \mathrm{sec}$; duty cycle, 20\%; SonoVue volume ratio, 1:5) were used. Subsequently, $0.2 \mathrm{ml}$ MTT $(0.5 \mathrm{mg} / \mathrm{ml})$ was added to the culture and incubated for an additional $4 \mathrm{~h}$ at $37^{\circ} \mathrm{C}$. The culture medium was then removed from the wells and replaced with $0.2 \mathrm{ml}$ dimethyl sulfoxide. Following agitation of the 96-well plates for 15-20 min on a swing bed, the absorbance was measured at a wavelength of $450 \mathrm{~nm}$ using a Model 680 Microplate Reader from Bio-Rad Laboratories (Hercules, CA, USA).

Statistical analysis. Student's t-test was utilized to identify the significance of differences between the experimental and control groups using SPSS, version 17.0 (SPSS, Inc., Chicago, IL, USA). $\mathrm{P}<0.05$ was considered to indicate a statistically significant difference. All experiments were conducted in triplicate.

\section{Results}

Characterization and drug-loading rate of $P T X-P L G A-m P E G$ $N C s$. The results from the particle size and $\zeta$ potential analyzer demonstrated that the PTX-PLGA-mPEG NC sizes were between 85.75 and $632.43 \mathrm{~nm}$; the average size was $276.38 \mathrm{~nm}$ and the $\zeta$ potential was $-6.94 \mathrm{mV}$ (Fig. 1). 

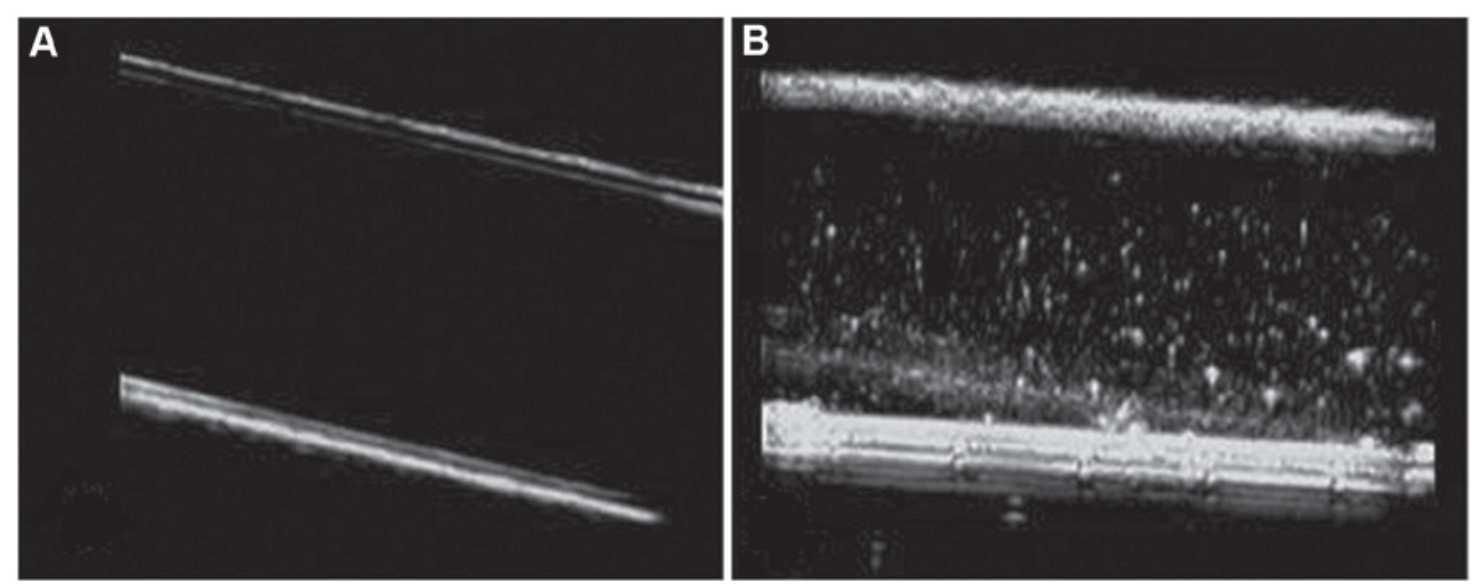

Figure 3. Ultrasound contrast-enhanced images in vitro. (A) Tube filled with degassed water; (B) tube filled with paclitaxel-poly(lactic-co-glycolic acid)-monomethoxy poly(ethylene glycol) nanocapsule solution.

A

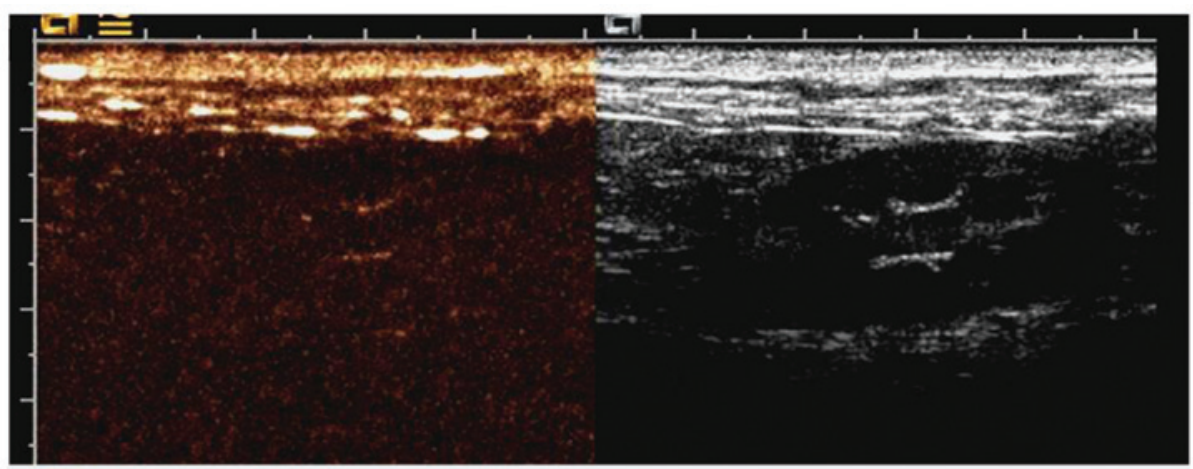

$\mathbf{B}$

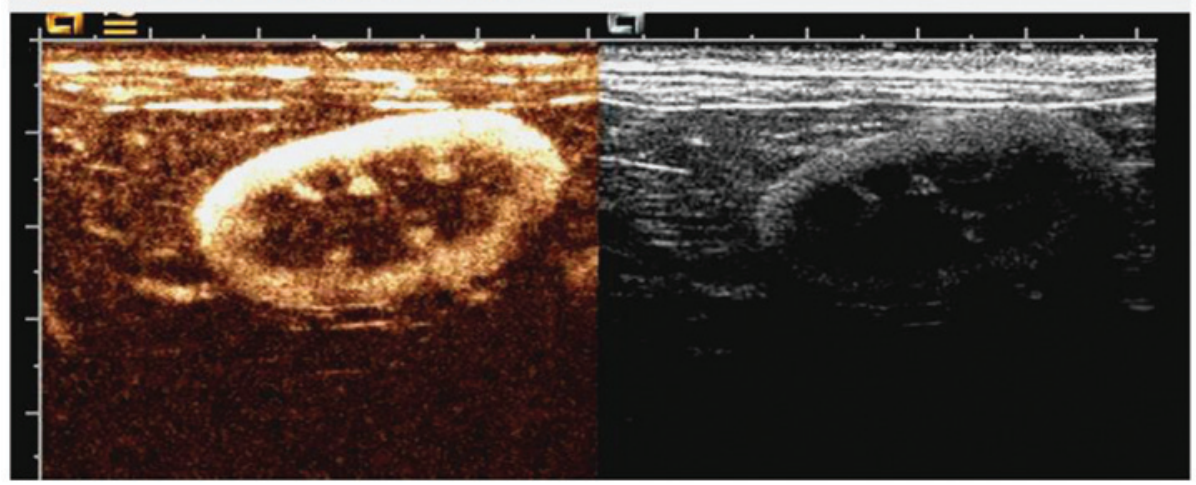

Figure 4. Representative in vivo ultrasound contrast-enhanced images in the rabbit right kidney. (A) Pre-and (B) post-administration of paclitaxel-poly(lactic-co-glycolic acid)-monomethoxy poly(ethylene glycol) nanocapsule solution. Left, contrast mode; right, conventional B-mode.

Observed by transmission electron microscopy (TEM; Olympus IX53; Olympus, Tokyo, Japan), the morphology of the PTX-PLGA-mPEG NCs were spherical with advanced dispersion and no aggregation. The inside of the PTX-NCs exhibited hollow honeycomb-like holes as observed in the TEM images shown in Fig. 2. The drug-loading rate of PTX-NCs was calculated to be $1.6 \%$.

US contrast-enhanced imaging of PTX-PLGA-mPEG NCs. Observed from US contrast-enhanced images, a tube filled with PTX-PLGA-mPEG NC UCA displayed a strong dottedecho, whereas a tube filled with degassed water was observed as black (Fig. 3). Imaging of the rabbit right kidney in vivo following PTX-PLGA-mPEG NC UCA administration resulted in excellent contrast-enhanced images, whilst unclear images were observed pre-administration (Fig. 4). However, the contrast-enhanced images of superficial pancreatic tumors in nude mice following administration of PTX-PLGA-mPEG NC UCA and SonoVue suspension were unclear, similar to the level of clarity prior to administration (Fig. 5).

Detection of cellular uptake of NCs by fluorescence microscopy. As presented in Fig. 6, greater fluorescence was observed in Aspc-1 cells $5 \mathrm{~h}$ subsequent administration of the Rh-PLGA-mPEG NC solution (panels N1-3), compared with the control groups [PBS (panels P1-3) and Rh only (panels R1-3)]. Furthermore, greater fluorescence was observed in Aspc-1 cells following administration of the Rh-PLGA-mPEG NC 
A

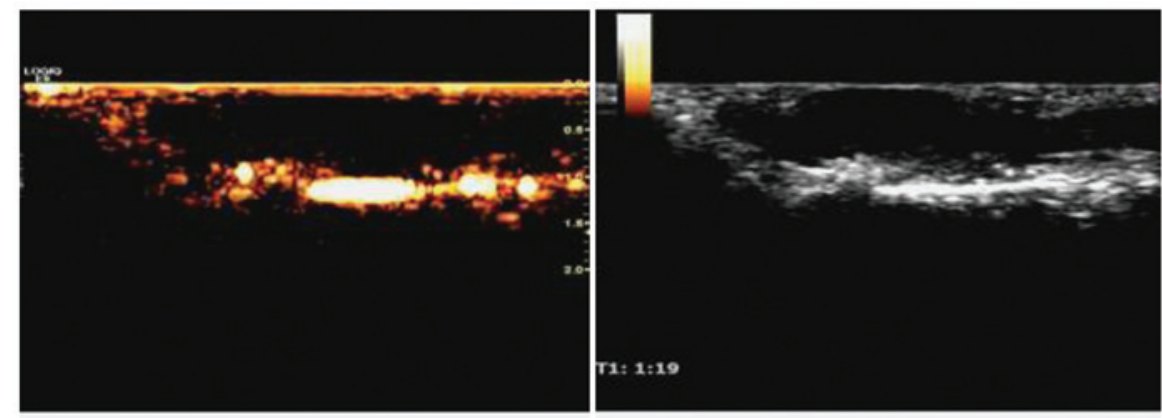

B

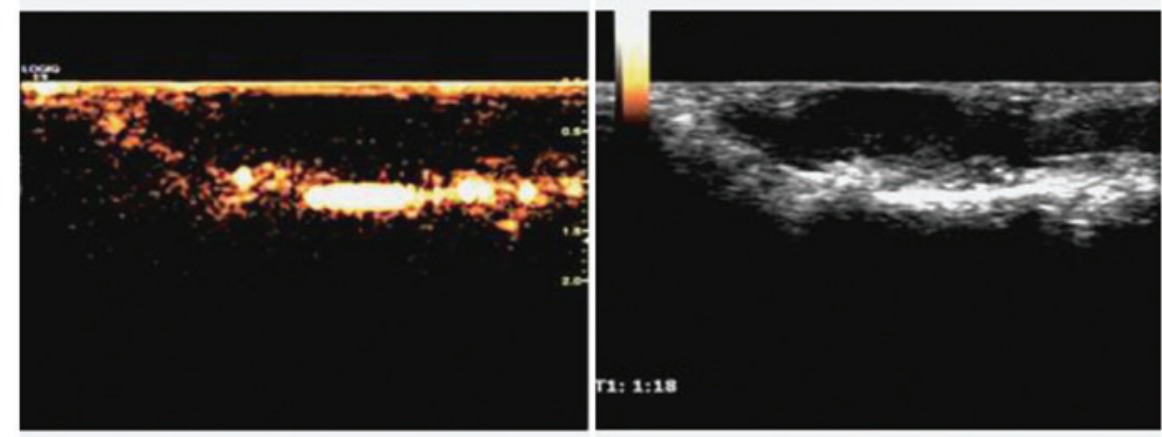

C

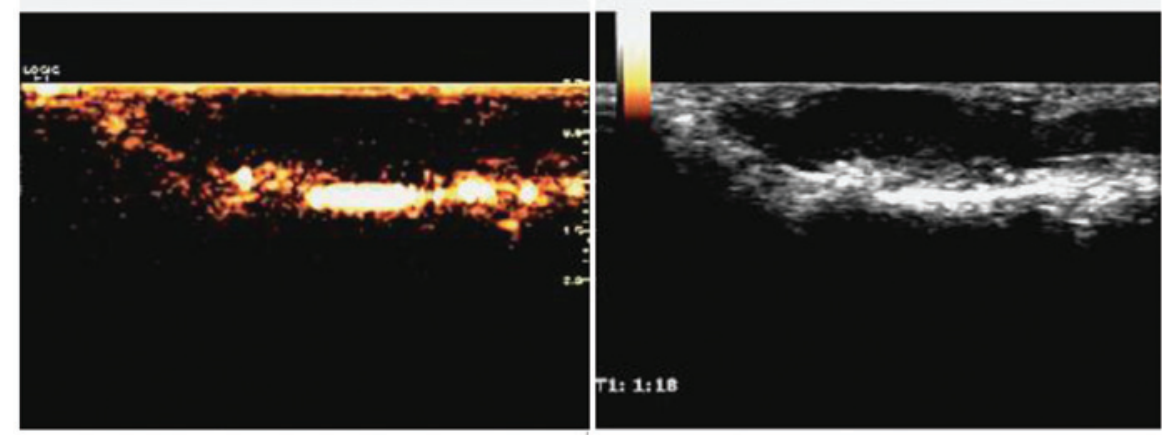

Figure 5. Representative in vivo ultrasound contrast-enhanced images of pancreatic superficial tumors in nude mice. (A) Pre- and (B) post-administration of paclitaxel-poly(lactic-co-glycolic acid)-monomethoxy poly(ethylene glycol) nanocapsule solution; and (C) post-administration of SonoVue. Left, contrast mode; right, conventional B-mode.

solution under the condition of UTMD (panel N3) compared with US (panel N2). No marked fluorescence was observed in the control groups (PBS and Rh only groups) under any of the conditions (panels P1-3 and R1-3).

Quantification of cellular uptake of NCs by flow cytometry. In the NCs + US and NCs + UTMD groups, the intracellular uptake rates were significantly greater than in the group with NCs alone $(\mathrm{P}<0.05)$. The NC uptake efficiency was not significantly higher in the NCs + UTMD group compared with that of the NCs + US group (Fig. 7)

MTT assay. The cell viabilities of Aspc-1 pancreatic cancer cells following administration of PBS, SonoVue, blank NCs, blank NCs + US, or blank NCs + UTMD for 24 or $48 \mathrm{~h}$ were all between 92 and 96\% (Fig. 8A). No significant differences in cell viability were identified between these groups $(\mathrm{P}>0.05)$. In the PTX-NC groups, it was identified that the NCs did not significantly affect cell viability in the absence of US or UTMD compared with PTX treatment alone $(\mathrm{P}>0.05)$. However, in the US or UTMD groups, following incubation for 24 or $48 \mathrm{~h}$, the PTX-NC cytotoxicities towards the cells were indicated to be significantly higher than those that underwent treatment with
PTX alone $(\mathrm{P}<0.05)$. Furthermore, subsequent to incubation for 24 or $48 \mathrm{~h}$, the cell viabilities of the pancreatic cancer cells with PTX-NCs mediated by UTMD were significantly lower than those mediated by US $(\mathrm{P}<0.05)$ (Fig. 8B).

\section{Discussion}

Conventional UCAs, such as the lipid-shelled SonoVue filled with sulfur hexafluoride gas, have been widely used in clinical practice. These types of UCAs have the ability to produce useful contrast-enhanced images, but cannot act therapeutically. It has been demonstrated that drugs and genes are able to adhere to the surface of the UCAs, or be encapsulated into them to be used therapeutically (12). The current study designed a novel nano-UCA that can be used not only in contrast-enhanced imaging, but also to deliver drugs into tumor cells.

This novel nano-UCA was made using PLGA-mPEG, a commonly used biodegradable polyester material, which has been approved to be nontoxic and harmless by the US Food and Drug Administration (13). An mPEG molecule can prolong the body circulation time of drugs and increase time at the tumor tissue by reducing their recognition by the reticuloendothelial system, thus an mPEG molecule may be beneficial in the 

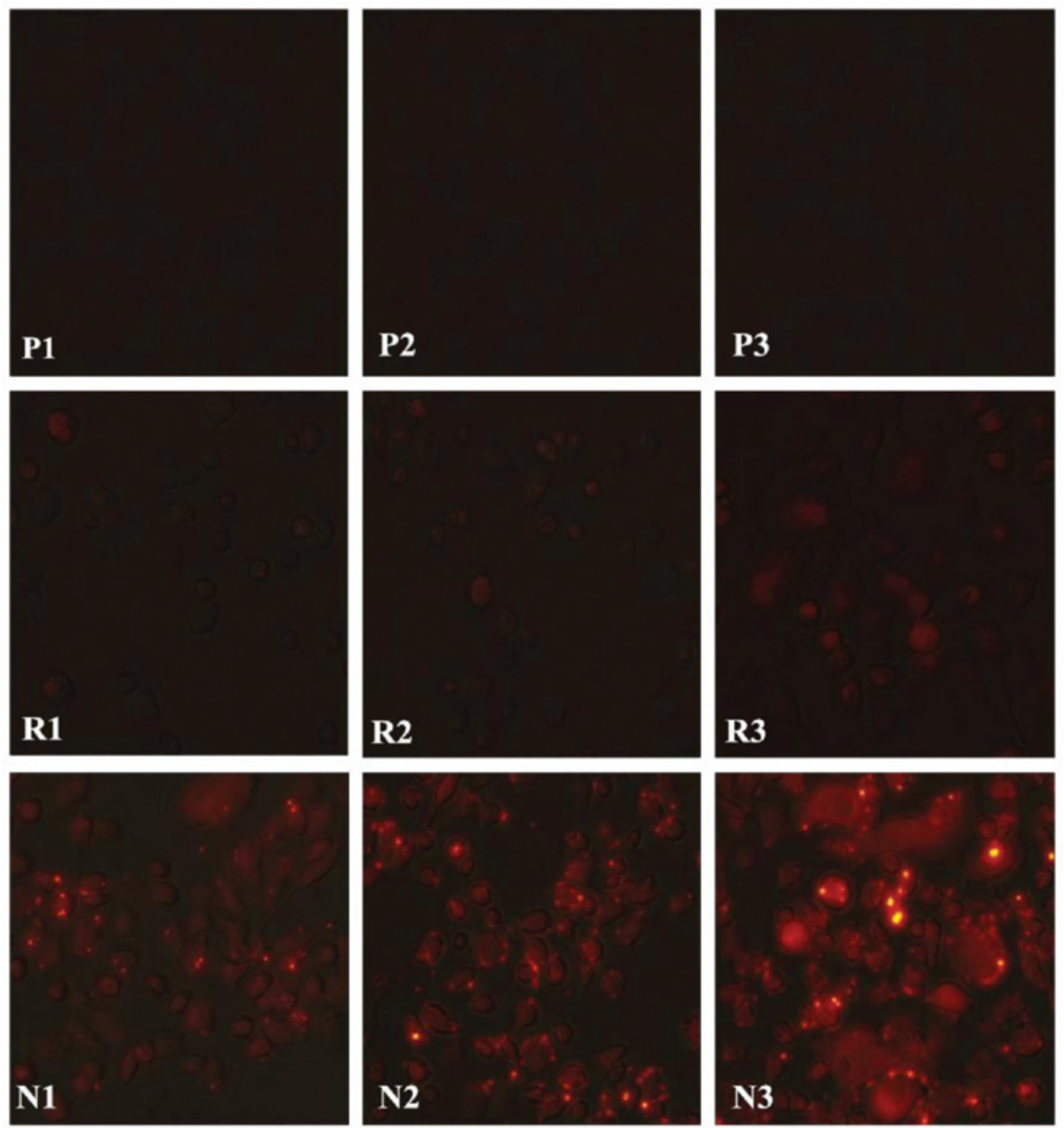

Figure 6. Intracellular uptake of Rh-PLGA-mPEG NCs following $5 \mathrm{~h}$ of different conditions. Representative images displaying the fluorescence of the 9 groups, observed under the fluorescence microscope. P, PBS group; R, Rh group; N, Rh-PLGA-mPEG NCs group. 1, no US; 2, with US; 3, with UTMD. Rh-PLGA-mPEG NCs, rhodamine-poly(lactic-co-glycolic acid)-monomethoxy poly(ethylene glycol) nanocapsules; PBS, phosphate-buffered saline; Rh, rhodamine; US, ultrasound; UTMD, US-targeted microbubble destruction.
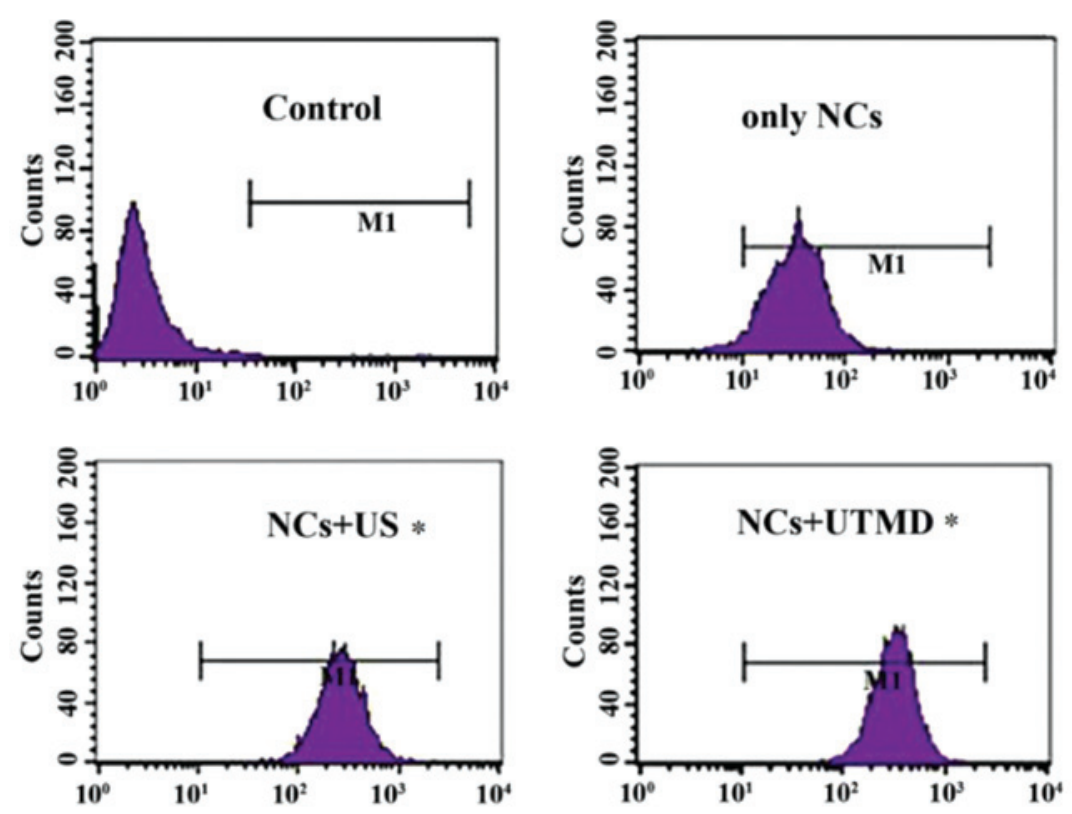

Figure 7. Flow cytometry results indicating the cell fluorescence of Aspc-1 cells in each group (controls, $0.05 \%$; NCs only, 23.42\%; NCs + US, $77.35 \%$; and NCs + UTMD, $81.47 \%) . P<0.05$ in the NC + US and NC + UTMD, compared with the only NC group. NCs, nanocapsules; US, ultrasound, UTMD, US-targeted microbubble destruction; M1, gate. 
A

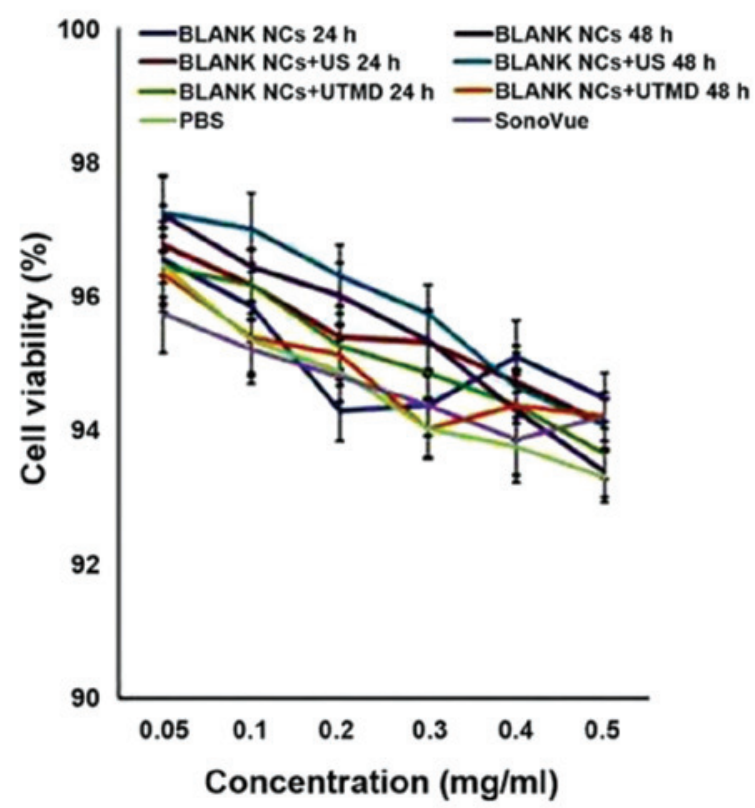

B

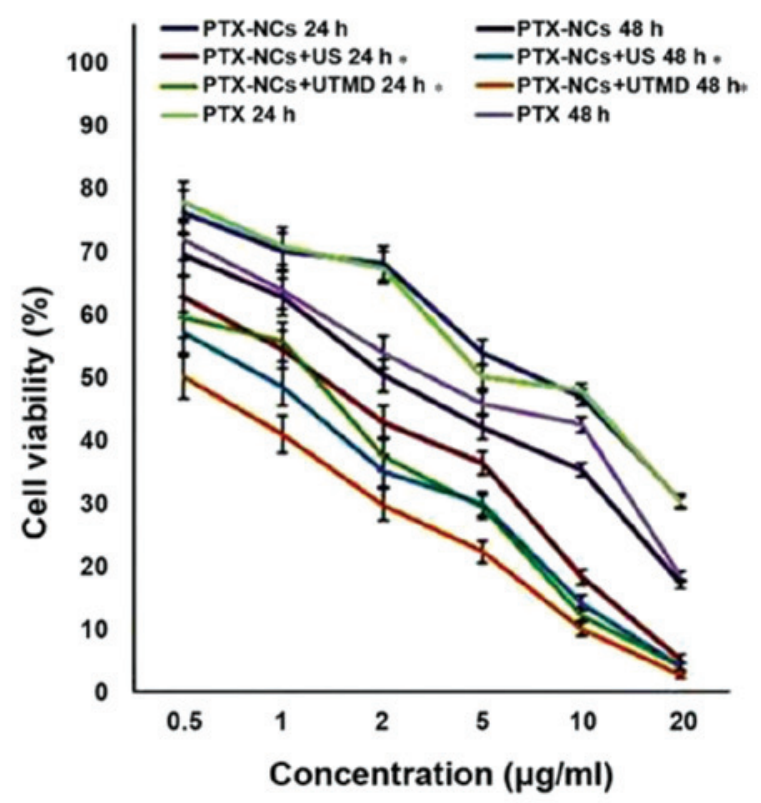

Figure 8. MTT assay. The cell viabilities of Aspc-1 pancreatic cancer cells 24 or 48 h subsequent to different treatments. (A) Blank nanocapsules; (B) paclitaxel-poly(lactic-co-glycolic acid)-monomethoxypoly (ethylene glycol) nanocapsules.

treatment of tumors in vivo. The double-emulsion method was used for the preparation of PTX-PLGA-mPEG NCs, which were observed by TEM to be spherical in shape, with a diameter ranging between 85.75 and $632.43 \mathrm{~nm}$. During the course of synthesis, the encapsulated water in the inner aqueous phase of the NCs was sublimated by lyophilization. This resulted in small hollow holes with a honeycomb structure, providing a basis for the US-responsive properties. The properties of PTX-PLGA-mPEG NCs following lyophilization were stable; this type of nano-UCA powder may be kept for a long time at a temperature of $-20^{\circ} \mathrm{C}$ without alteration to its appearance. Furthermore, the morphology observed by TEM following resolution revealed good dispersion and no aggregation.

It was also observed that PTX-PLGA-mPEG NCs yielded effective contrast-enhanced images in vitro and in rabbit right kidney in vivo. However, contrast images in superficial pancreatic cancer tumors in nude mice were not satisfactory with administration of PTX-PLGA-mPEG NCs or with SonoVue solution. A potential explanation may be as follows: In general, the vessels of the tumor were divided into two sections; one section consisted of the original vessels, the endothelial gaps of which were $<100 \mathrm{~nm}$. The other section was composed of newly formed vessels, the endothelial structure of which was not intact and did not contain smooth muscle. Due to this, the endothelial gaps in these newly formed blood vessels were larger; between 380-780 nm (14). As a result of this, a greater nano-UCA influx into the tumor tissues may have occurred, compared with micro-UCAs. The pancreatic tumor tissues of nude mice and humans presented specific pathological and anatomical similarities, with dense, poorly vascularized connective tissues immersed in a large volume of fibrous tissues and lymphocytes (15). Hence, to a certain extent, nano-UCAs may produce improved contrast-enhanced images in rabbit kidney and maintain a sufficient blood supply, compared with those in superficial tumors of nude mouse pancreas. Additionally, the hollow holes in lyophilized PTX-PLGA-mPEG NCs may be so small that they lead to strong ultrasonic reflection, and a higher concentration of the nano-UCA solution would be required for US contrast imaging in pancreatic tumors.

The present study demonstrated a greater Rh-PLGA-mPEG NC uptake (red fluorescent signals in Fig. 6) in Aspc-1 cells following administration of Rh-PLGA-mPEG NCs mediated by US or UTMD (N2 and 3), when comparing intracellular uptake ratios among the PBS, Rh alone and Rh-PLGA-mPEG groups in US (N1), US (N1) and UTMD (N3) conditions. Furthermore, stronger red fluorescent signals in Aspc-1 cells were observed following administration of the Rh-PLGA-mPEG NCs with UTMD (N3) compared with US (N2). Similar results were observed when measuring $\mathrm{NC}$ cellular uptake with flow cytometry. The cellular uptake efficiencies of the PLGA-mPEG NC groups with US and UTMD were higher than the uptake of the PLGA-mPEG NCs alone. The PLGA-mPEG NC cellular uptake efficiency in the PLGA-mPEG NCs + UTMD group was not significantly higher than in the PLGA-mPEG NCs + US group. These results indicate that US and UTMD are effective driving forces that may be favorable methods to increase PLGA-mPEG NC uptake to Aspc-1 pancreatic cancer cells.

Possible mechanisms for promoting NP uptake into cells have been studied, but the most efficient mechanism remains unclear: Micro-circumflex or micro-fluid generation by UTMD punching transient holes in the cell membrane surface (16); an increase in reactive oxygen species in cells; cell membrane transport abilities becoming activated; and an increase in cell membrane temperature during US (17-21) are a number of possibilities. Furthermore, a previous study proposed a novel mechanism, that UTMD may stimulate cellular clathrin-dependent endocytosis (22).

In the MTT assay, the cell viabilities of Aspc-1 pancreatic cancer cells following administration of PBS, SonoVue 
or blank PLGA-mPEG NCs with or without US or UTMD for 24 or $48 \mathrm{~h}$ were all above $90 \%$. This demonstrated that the cellular cytotoxicity of the blank PLGA-mPEG NCs at each concentration tested was negligible, and also that the optimal US and UTMD conditions had almost no effect on these Aspc-1 cells in vitro without the PTX-PLGA-mPEG NCs. However, when combined with PTX-PLGA-mPEG NCs, the cytotoxicity was greater following US and UTMD than with no US. In addition, due to more powerful sonoporation, UTMD elicited an increased NC uptake into the Aspc-1 cells compared with US. Thus, US and UTMD were demonstrated to be powerful physical techniques, which may safely and efficiently deliver PTX-PLGA-mPEG NCs into Aspc-1 cells in vitro, consequently producing an antitumorigenic effect.

In conclusion, a novel PTX-PLGA-mPEG NC technique, which combined US contrast imaging and antitumor therapy, was successfully designed and prepared. UTMD, a promising physical targeting vehicle, may facilitate improved PTX-PLGA-mPEG NC uptake into Aspc-1 pancreatic cancer cells and enhanced antitumorigenic action in vitro. Thus, the combination of nanotechnology and US may present a novel method for monitoring and treating tumors. Further study is required to continue the investigation of US-specific contrast imaging and antitumor treatment, and future studies should include a variety of tumor types in vivo.

\section{Acknowledgements}

The current study was supported by the Department of Ultrasound, Shanghai First People's Hospital Affiliated to Shanghai Jiao tong University School of Medicine (Shanghai, China) and the National Natural Science Foundation of China. Project approval nos. 81271596 and 81171352.

\section{References}

1. Xing Z, Ke H, Wang J, Zhao B, Yue X, Dai Z and Liu J: Novel ultrasound contrast agent based on microbubbles generated from surfactant mixtures of Span 60 and polyoxyethylene 40 stearate. Acta Biomater 6: 3542-3549, 2010.

2. Chen M, Cooper HM, Zhou JZ, et al: Reduction in the size of layered double hydroxide nanoparticles enhances the efficiency of siRNA delivery. J Colloid Interface Sci 390: 275-281, 2013.

3. Du J, Shi QS, Sun Y, et al: Enhanced delivery of monomethoxypoly(ethylene glycol)-poly(lactic-co-glycolic acid)-poly l-lysine nanoparticles loading platelet-derived growth factor $\mathrm{BB}$ small interfering RNA by ultrasound and/or microbubbles to rat retinal pigment epithelium cells. J Gene Med 13: 312-323, 2011.

4. Fields RJ, Cheng CJ, Quijano E, et al: Surface modified poly $(\beta$ amino ester)-containing nanoparticles for plasmid DNA delivery. J Control Release 164: 41-48, 2012.
5. Lee SH, Bae KH, Kim SH, Lee KR and Park TG: Amine-functionalized gold nanoparticles as non-cytotoxic and efficient intracellular siRNA delivery carriers. Int J Pharm 364: 94-101, 2008

6. Arya G, Vandana M, Acharya S and Sahoo SK: Enhanced antiproliferative activity of Herceptin (HER2)-conjugated gemcitabine-loaded chitosan nanoparticle in pancreatic cancer therapy. Nanomedicine 7: 859-870, 2011.

7. Chappell JC, Song J, Burke CW, Klibanov AL and Price RJ: Targeted delivery of nanoparticles bearing fibroblast growth factor- 2 by ultrasonic microbubble destruction for therapeutic arteriogenesis. Small 4: 1769-1777, 2008.

8. Vancraeynest D, Havaux X, Pouleur AC, et al: Myocardial delivery of colloid nanoparticles using ultrasound-targeted microbubble destruction. Eur Heart J 27: 237-245, 2006.

9. Lin CY, Liu TM, Chen CY, et al: Quantitative and qualitative investigation into the impact of focused ultrasound with microbubbles on the triggered release of nanoparticles from vasculature in mouse tumors. J Control Release 146: 291-298, 2010.

10. Chumakova OV, Liopo AV, Andreev VG, et al: Composition of PLGA and PEI/DNA nanoparticles improves ultrasound-mediated gene delivery in solid tumors in vivo. Cancer Lett 261: 215-225, 2008.

11. Liu P, Qin L, Wang Q, et al: cRGD-functionalized mPEG-PLGA-PLL nanoparticles for imaging and therapy of breast cancer. Biomaterials 33: 6739-6747, 2012.

12. Liu Y, Miyoshi H and Nakamura M: Nanomedicine for drug delivery and imaging: a promising avenue for cancer therapy and diagnosis using targeted functional nanoparticles. Int J Cancer 120: 2527-2537, 2007.

13. Zhang Y, Chan HF and Leong KW: Advanced materials and processing for drug delivery: the past and the future. Adv Drug Deliv Rev 65: 104-120, 2013.

14. Oeffinger BE and Wheatley MA: Development and characterization of a nano-scale contrast agent. Ultrasonics 42: 343-347, 2004.

15. Wei JM, Xu XJ, Wang XY, et al: Potential relationship between pancreatic histological features and its diseases. Chin J Hepatobil Surg 14: 414-416, 2008 (In Chinese).

16. Prentice P, Cuschieri A, Dholakia K, et al: Membrane disruption by optically controlled microbubble cavitation. Nat Phys 1: 107-110, 2005.

17. Tachibana K, Uchida $\mathrm{T}$, Ogawa $\mathrm{K}$, et al: Induction of cell-membrane porosity by ultrasound. Lancet 353: 1409, 1999.

18. Van Wamel A, Kooiman K, Harteveld M, et al: Vibrating microbubbles poking individual cells: drug transfer into cells via sonoporation. J Control Release 112: 149-155, 2006.

19. Juffermans LJ, Dijkmans PA, Musters RJ, et al: Transient permeabilization of cell membranes by ultrasound-exposed microbubbles is related to formation of hydrogen peroxide. Am J Physiol Heart Circ Physiol 291: H1595-H1601, 2006.

20. Miller DL and Gies RA: The interaction of ultrasonic heating and cavitation in vascular bioeffects on mouse intestine. Ultrasound Med Biol 24: 123-128, 1998.

21. Schlicher RK, Radhakrishna H, Tolentino TP, et al: Mechanism of intracellular delivery by acoustic cavitation. Ultrasound Med Biol 32: 915-924, 2006.

22. Jin LF, Li F, Wang HP, Wei F, Qin P and Du LF: Ultrasound targeted microbubble destruction stimulates cellular endocytosis in facilitation of adeno-associated virus delivery. Int J Mol Sci 14: 9737-9750, 2013. 